\title{
TWO-DIMENSIONAL SIGNATURES
}

\author{
ILYA GERTSBAKH, ${ }^{*}$ Ben Gurion University \\ YOSEPH SHPUNGIN, ${ }^{* *}$ Shamoon College of Engineering \\ FABIO SPIZZICHINO, ${ }^{* * *}$ Sapienza University of Rome
}

\begin{abstract}
The notion of the signature is a basic concept and a powerful tool in the analysis of networks and reliability systems of binary type. An appropriate definition of this concept has recently been introduced for systems that have $v$ possible states (with $v \geq 3$ ). In this paper we analyze in detail several properties and the most relevant aspects of such a general definition. For simplicity's sake, we focus our attention on the case $v=3$. Our analysis will however provide a number of hints for understanding the basic aspects of the general case.
\end{abstract}

Keywords: Two-dimensional anchor; two-dimensional signature; G-series and G-parallel systems; multistate recurrent system; homogeneous polynomial

2010 Mathematics Subject Classification: Primary 60K10

Secondary 90B25

\section{Introduction}

The notion of the signature, as introduced by Samaniego [16], relates to binary coherent systems composed of binary components. This notion is becoming a core concept and a powerful tool in the field of reliability, and its extension to the case of multistate systems could therefore constitute a promising issue with important implications both in theoretical studies and computational analysis.

An appropriate definition of the signature for multistate systems has recently been introduced in [12] where some basic aspects have been analyzed, by maintaining the focus on the assumption of binary components; see also [8] and [9].

Let us denote by $v$ the number of possible states of the system and set $d=v-1$. For a binary system (i.e. in the case $d=1$ ), the signature, purely seen as a mathematical object, is nothing other than a probability distribution for a scalar $\{1, \ldots, n\}$-valued random variable, where $n$ is the number of components. It turns out that in the case of systems with $v>2$ possible states (and still containing $n$ binary components), the signature is a joint probability distribution for $d$ ordered, $\{1, \ldots, n\}$-valued random variables. Thus, $d$ can be interpreted as a dimension for the signature and, in the following, we will sometimes refer to the $v=2$ and $v>2$ cases as the univariate and the multivariate cases, respectively.

In a natural way, examples of applications of the multivariate signature can arise, e.g. in the field of network reliability: it is natural to consider the disintegration of an initially connected

\footnotetext{
Received 9 August 2011; revision received 31 October 2011.

* Postal address: Department of Mathematics, Ben Gurion University, Shai Agnon 30/25, 69362, Tel-Aviv, Israel. Email address: elyager@bezeqint.net

** Postal address: Deptartment of Software Engineering, Shamoon College of Engineering, Bialik/Basel Streets, Beer Sheva, Israel. Email address: yosefs@sce.ac.il

*** Postal address: Deptartment of Mathematics, Sapienza University of Rome, Piazzale Aldo Moro 2, 00185, Rome, Italy. Email address: fabio.spizzichino@uniroma1.it
} 
network into isolated parts when its edges fail in a random order. As suggested in [12], state $v$ corresponds to the situation of complete connection, state $v-1$ corresponds to the network with two isolated parts, ..., and state 0 corresponds to the presence of $v$ or more isolated parts.

The concept of the multivariate signature can therefore have important applications; furthermore, it is related to interesting combinatorial meanings. For these reasons, different aspects of this concept are worth detailing further.

Concerning the univariate notion, the following aspects have a basic role.

(a) The signature can be equivalently defined as a purely combinatorial characteristic of a system (without any reference to the probability law of the components' lifetimes) or as a probability distribution associated to the assumption that the lifetimes are independent and identically distributed (i.i.d.) or, more generally, exchangeable.

(b) In the i.i.d. case we can express the system reliability at a time $t$ in terms of the signature and of the survival functions of lifetimes' order statistics. In this respect, we can derive a fundamental formula that is obtained as a simple consequence of the rule of total probabilities and that is at the basis of the use of signatures in system reliability.

For more details on these issues, see, in particular, [5], [6], [7, Chapter 6], [11], [15], [16], [17, Chapter 3], [18], [19], and the references cited therein.

Concerning the case of multistate systems, we can in particular anticipate that the above aspects still hold, provided that appropriate multivariate reformulations are made.

The present paper is specifically devoted to the analysis of systems with three different states (i.e. we consider the case in which $v=3$ and $d=2$ ). Such a case can in fact be of special interest in reliability analysis and is still feasibly tractable, at a time. For these systems, we aim to detail the fundamental properties of the notion of the (bivariate) signature. Our analysis also aims to provide some general clues for the cases where $v>3$.

We will also consider systems obtained as generalized series and generalized parallels of a pair of three-state modules. For these systems, we show results that properly extend what has been obtained in [10] for parallel and series systems of two separate binary modules, namely that simple formulae hold for computing the signature of systems that can be decomposed as series or parallel connections of two separate subsystems. The reliability formula mentioned in (b) has been used in [10] as a fundamental tool. We shall see here that an analogous formula holds for the multivariate case and that the latter is at the basis of the computation of the bivariate signature of generalized parallel and series systems. Moreover, it emerged in [10] that objects of central interest are actually the notions of cumulative signature and tail (or survival) signature. We shall see that a completely similar situation is encountered in the multivariate case as well.

A further issue, emerging from the analysis developed in [10] for $v=2$, concerns the construction of pairs of complex systems sharing the same signature. This construction can also be easily extended to the multivariate case.

The paper has the following structure. In Section 2 we study the basic aspects of the (bivariate) signature of a three-state system. In particular, we provide a formula that relates the signature to the system's reliability function. Furthermore, we give the combinatorial meaning of the cumulative notions of the signature. In Section 3 we consider three-state systems that are obtained from generalized series and generalized parallel connections of separate three-state modules; we actually obtain their signature in terms of the signatures of the modules. Finally, Section 4 will be devoted to a discussion containing some examples and general remarks about signatures and related concepts. 


\section{Notation and basic aspects of bivariate signatures}

We consider a three-stage monotone system containing $n$ binary components and characterized by its structure function

$$
\varphi:\{0,1\}^{n} \rightarrow\{0,1,2\}
$$

where 2 is the perfection state, 0 is a complete failure state, and 1 is a state of partial failure for the system. Concerning this system, we make the following assumptions.

(A1) The system is coherent in the sense that $\varphi$ is increasing and each component is relevant.

This assumption is a direct extension of the notion of coherence for binary systems (see [1]). Each component being relevant means that, for each index $i \in\{1, \ldots, n\}$, there exists a binary vector $\left(x_{1}, \ldots, x_{i-1}, x_{i+1}, \ldots, x_{n}\right)$ such that

$$
\varphi\left(x_{1}, \ldots, x_{i-1}, 1, x_{i+1}, \ldots, x_{n}\right)-\varphi\left(x_{1}, \ldots, x_{i-1}, 0, x_{i+1}, \ldots, x_{n}\right)=1 .
$$

(A2) Let $x=\left(x_{1}, \ldots, x_{n}\right)$ and $y=\left(y_{1}, \ldots, y_{n}\right) \in\{0,1\}^{n}$ be such that, for one index $i \in\{1, \ldots, n\}, x_{i}=1, y_{i}=0$, and $x_{j}=y_{j}$ for $j \neq i$. Then

$$
\varphi(x)-\varphi(y) \leq 1 \text {. }
$$

In words, (A2), the regularity condition, requires that the failure of one component either does not change the state of the system or, at most, it changes the state by one unit. For a network, this is true if (i) the components are the edges and the network's state is the number of isolated components in it and (ii) the components are the edges and the network's state decreases step by step as the number of isolated parts in the system increase. This property however no longer holds when, for example, in case (i) the prone-to-failure components are the network's nodes and the failure of a node amounts to erasing all edges incident to it, and in case (ii) the prone-to-failure components are the network's nodes and the failure of a node amounts to erasing all edges incident to it.

We now introduce some convenient notation, define the concept of the (bivariate) signature of $\varphi$, and detail some related bivariate counterparts of the basic properties of the univariate signature given in the literature.

Denote by $\mathcal{P}_{n}$ the set of permutations of $\{1,2, \ldots, n\}$. For $\pi \equiv(\pi(1), \ldots, \pi(n)) \in \mathcal{P}_{n}$ and $x \equiv\left(x_{1}, \ldots, x_{n}\right) \in\{0,1\}^{n}$, let $x \circ \pi \in\{0,1\}^{n}$ denote the vector whose $\pi$ (1)th coordinate is $x_{1}, \pi(2)$ th coordinate is $x_{2}$, etc. To any $\pi \in \mathcal{P}_{n}$, we respectively associate the integers $m_{1}=m_{1}(\pi)$ and $m_{2}=m_{2}(\pi)$ such that

$$
\begin{aligned}
& \varphi\left(x_{1}^{\prime} \circ \pi\right)=2, \quad \varphi\left(x_{1}^{\prime \prime} \circ \pi\right)=1, \\
& \varphi\left(x_{2}^{\prime} \circ \pi\right)=1, \quad \varphi\left(x_{2}^{\prime \prime} \circ \pi\right)=0,
\end{aligned}
$$

where

$$
\begin{aligned}
x_{1}^{\prime} & \equiv\left(0_{1}, 0_{2}, \ldots, 0_{m_{1}-1}, 1_{m_{1}}, 1_{m_{1}+1}, \ldots, 1_{n}\right), \\
x_{1}^{\prime \prime} & \equiv\left(0_{1}, 0_{2}, \ldots, 0_{m_{1}-1}, 0_{m_{1}}, 1_{m_{1}+1}, \ldots, 1_{n}\right), \\
x_{2}^{\prime} & \equiv\left(0_{1}, 0_{2}, \ldots, 0_{m_{2}-1}, 1_{m_{2}}, 1, \ldots, 1\right), \\
x_{2}^{\prime \prime} & \equiv\left(0_{1}, 0_{2}, \ldots, 0_{m_{2}-1}, 0_{m_{2}}, 1_{m_{2}+1}, \ldots, 1_{n}\right) .
\end{aligned}
$$

For $1 \leq l<r \leq n$, consider the subsets

$$
A_{l, r}:=\left\{\pi \in \mathcal{P}_{n} \mid m_{1}(\pi)=l, m_{2}(\pi)=r\right\} .
$$

Obviously, these sets form a partition of $\mathcal{P}_{n}$ that is determined by the structure function $\varphi$. 
Definition 1. The signature of $\varphi$ is defined as the $(n \times n)$-dimensional matrix with elements

$$
s_{l, r}= \begin{cases}\frac{\left|A_{l, r}\right|}{n !} & \text { for } l<r, \\ 0 & \text { otherwise }\end{cases}
$$

where $\left|A_{l, r}\right|$ denotes the cardinality of $A_{l, r}$.

This definition of the (bivariate) signature is a purely combinatorial definition; see also [12]. Note that we completely avoided any reference to lifetimes of components and their probability law.

Now we consider the (random) component lifetimes $T_{1}, \ldots, T_{n}$ and denote by $N_{t}$ the number of component failures observed up to time $t(t>0)$ :

$$
N_{t}:=\sum_{j=1}^{n} \mathbf{1}_{\left\{T_{j} \leq t\right\}} .
$$

Furthermore, we respectively denote by $M_{1}$ and $M_{2}$ the (random) anchors for the first-level and second-level degradations of the system. Namely, $M_{1}$ is the number of component failures until the exit from state 2 into state 1 and $M_{2}$ is the number of component failures until the exit from state 1 into state 0 (i.e. until the system failure).

In order to define $M_{1}$ and $M_{2}$ more formally, we denote by $\Pi$ the random permutation of $\{1,2, \ldots, n\}$ determined by the order of component failures.

Definition 2. Define the random variables $M_{1}$ and $M_{2}$ by

$$
M_{1}:=m_{1}(\Pi), \quad M_{2}:=m_{2}(\Pi) .
$$

We now restrict our attention to the case when $T_{1}, \ldots, T_{n}$ are i.i.d. random variables with a continuous probability distribution $G$. In this case, for the random permutation $\Pi$, we have

$$
\mathrm{P}(\Pi=\pi)=\frac{1}{n !} \quad \text { for all } \pi \in \mathcal{P}_{n} .
$$

We also have the probabilities

$$
\mathrm{P}\left(M_{1}=l, M_{2}=r\right):=\mathrm{P}\left(\Pi \in A_{l, r}\right),
$$

from which we immediately obtain the following result.

Lemma 1. It holds that

$$
\mathrm{P}\left(M_{1}=l, M_{2}=r\right)=s_{l, r}, \quad 1 \leq l<r \leq n .
$$

Remark 1. Lemma 1 is the analogue, for the present bivariate case, of a basic, well-known fact valid for the signatures of common two-state systems: it shows that the combinatorial notion of the signature can immediately be given a probabilistic meaning by considering the case of systems with i.i.d. component lifetimes.

Let $\Upsilon_{1}$ and $\Upsilon_{2}$ be the calendar times to the first and second degradation steps, respectively. More formally, define $\Upsilon_{1}$ and $\Upsilon_{2}$ as

$$
\Upsilon_{1}:=\inf \left\{t>0 \mid N_{t}=M_{1}\right\}, \quad \Upsilon_{2}:=\inf \left\{t>0 \mid N_{t}=M_{2}\right\} .
$$


For any fixed pair $\left(\xi_{1}, \xi_{2}\right)$ with $0<\xi_{1}<\xi_{2}$, we want to find an expression for $\mathrm{P}\left(\Upsilon_{1}>\xi_{1}\right.$, $\Upsilon_{2}>\xi_{2}$ ). To this end, we derive a relation between the joint distribution of $\Upsilon_{1}$ and $\Upsilon_{2}$ and the bivariate signature (2).

Note that (obviously), for any $\xi>0$,

$$
\mathrm{P}\left(\Upsilon_{1}>\xi \mid M_{1}=l, N_{\xi}=n\right)= \begin{cases}1 & \text { if } n<l \\ 0 & \text { if } n \geq l\end{cases}
$$

Similarly,

$$
\mathrm{P}\left(\Upsilon_{2}>\xi \mid M_{2}=r, N_{\xi}=n\right)= \begin{cases}1 & \text { if } n<r \\ 0 & \text { if } n \geq r .\end{cases}
$$

Denoting by $E\left(l, r, n_{1}, n_{2}\right)$ the event

$$
E\left(l, r, n_{1}, n_{2}\right):=\left(M_{1}=l, N_{\xi_{1}}=n_{1}, M_{2}=r, N_{\xi_{2}}=n_{2}\right),
$$

we can then write, for $l<r, n_{1}<n_{2}$,

$$
\mathrm{P}\left(\Upsilon_{1}>\xi_{1}, \Upsilon_{2}>\xi_{2} \mid E\left(l, r, n_{1}, n_{2}\right)\right)=\mathbf{1}_{\left\{n_{1}<l\right\}} \mathbf{1}_{\left\{n_{2}<r\right\}} .
$$

Furthermore, as an immediate consequence of the assumption that $T_{1}, \ldots, T_{n}$ are i.i.d. with distribution $G(t)$, we have the following result.

Lemma 2. It holds that

$$
\begin{aligned}
\mathrm{P}\left(N_{\xi_{1}}=n_{1}, N_{\xi_{2}}=n_{2}\right)= & \frac{n !}{n_{1} !\left(n_{2}-n_{1}\right) !\left(n-n_{2}\right) !} \\
& \times\left[G\left(\xi_{1}\right)\right]^{n_{1}}\left[G\left(\xi_{2}\right)-G\left(\xi_{1}\right)\right]^{n_{2}-n_{1}}\left[1-G\left(\xi_{2}\right)\right]^{n-n_{2}} .
\end{aligned}
$$

Lemma 3. The pairs $\left(M_{1}, M_{2}\right)$ and $\left(N_{\xi_{1}}, N_{\xi_{2}}\right)$ are stochastically independent.

Lemma 2 is obvious. The proof of Lemma 3 is also rather immediate. In fact, in view of Lemma 1 , we can say that $T_{1}, \ldots, T_{n}$ being i.i.d. renders the random permutation $\Pi$ independent of $\left(T_{1}, \ldots, T_{n}\right)$. Thus, $\left(M_{1}, M_{2}\right)$ and $\left(N_{\xi_{1}}, N_{\xi_{2}}\right)$ are independent, since $\left(M_{1}, M_{2}\right)$ is a function of $\Pi$ and $\left(N_{\xi_{1}}, N_{\xi_{2}}\right)$ is a function of $\left(T_{1}, \ldots, T_{n}\right)$.

Obviously, $\mathrm{P}\left(E\left(l, r, n_{1}, n_{2}\right)\right)=0$ whenever $l \geq r$ and/or $n_{1} \geq n_{2}$. Otherwise, for $1 \leq l<r$ and $n_{1}<n_{2}$,

$$
\begin{aligned}
\mathrm{P}\left(E\left(l, r, n_{1}, n_{2}\right)\right)= & \mathrm{P}\left(M_{1}=l, M_{2}=r\right) \mathrm{P}\left(N_{\xi_{1}}=n_{1}, N_{\xi_{2}}=n_{2}\right) \\
= & s_{l, r} \frac{n !}{n_{1} !\left(n_{2}-n_{1}\right) !\left(n-n_{2}\right) !} \\
& \times\left[G\left(\xi_{1}\right)\right]^{n_{1}}\left[G\left(\xi_{2}\right)-G\left(\xi_{1}\right)\right]^{n_{2}-n_{1}}\left[1-G\left(\xi_{2}\right)\right]^{n-n_{2}} .
\end{aligned}
$$

Let us now consider the 'bivariate tail signature' as the matrix $\bar{S}$ with elements defined by

$$
\begin{aligned}
\bar{S}_{n_{1}, n_{2}} & =\mathrm{P}\left(M_{1}>n_{1}, M_{2}>n_{2}\right) \\
& =\sum_{l=n_{1}+1}^{n-1} \sum_{r=\min \left(n_{2}+1, l+1\right)}^{n} \mathrm{P}\left(M_{1}=l, M_{2}=r\right) \\
& =\sum_{l=n_{1}+1}^{n-1} \sum_{r=\min \left(n_{2}+1, l+1\right)}^{n} s_{l, r} .
\end{aligned}
$$

We can now conclude with the following. 
Theorem 1. Let

$$
u=G\left(\xi_{1}\right), \quad v=G\left(\xi_{2}\right)-G\left(\xi_{1}\right), \quad z=1-G\left(\xi_{2}\right) .
$$

Then

$$
\mathrm{P}\left(\Upsilon_{1}>\xi_{1}, \Upsilon_{2}>\xi_{2}\right)=\sum_{n_{1}=0}^{n-1} \sum_{n_{2}=n_{1}+1}^{n} u^{n_{1}} v^{n_{2}-n_{1}} z^{n-n_{2}} \bar{S}_{n_{1}, n_{2}} \frac{n !}{n_{1} !\left(n_{2}-n_{1}\right) !\left(n-n_{2}\right) !} .
$$

Proof. We have

$$
\begin{aligned}
\mathrm{P}\left(\Upsilon_{1}\right. & \left.>\xi_{1}, \Upsilon_{2}>\xi_{2}\right) \\
& =\sum_{l<r} \sum_{n_{1}<n_{2}} \mathrm{P}\left(\Upsilon_{1}>\xi_{1}, \Upsilon_{2}>\xi_{2} \mid E\left(l, r, n_{1}, n_{2}\right)\right) \mathrm{P}\left(E\left(l, r, n_{1}, n_{2}\right)\right) \\
& =\sum_{n_{1}<n_{2}} \sum_{l=n_{1}+1}^{n-1} \sum_{r=\min \left(n_{2}+1, l+1\right)}^{n} \mathrm{P}\left(E\left(l, r, n_{1}, n_{2}\right)\right) \\
& =\sum_{n_{1}<n_{2}} \sum_{l=n_{1}+1}^{n-1} \sum_{r=\min \left(n_{2}+1, l+1\right)}^{n} \mathrm{P}\left(N_{\xi_{1}}=n_{1}, N_{\xi_{2}}=n_{2}\right) \mathrm{P}\left(M_{1}=l, M_{2}=r\right) \\
& =\sum_{n_{1}<n_{2}} \mathrm{P}\left(N_{\xi_{1}}=n_{1}, N_{\xi_{2}}=n_{2}\right)\left(\sum_{l=n_{1}+1}^{n-1} \sum_{r=\min \left(n_{2}+1, l+1\right)}^{n} s_{l, r}\right),
\end{aligned}
$$

whence (5) readily follows, by taking into account Lemma 2 and relation (4).

Remark 2. Equation (5) expresses the joint survival function of the degradation times in the form of a homogeneous polynomial of degree $n$ in three variables, $u, v$, and $z$, where the tail signature probabilities $\bar{S}_{n_{1}, n_{2}}$ appear in the coefficients. This formula is a bivariate analogue of the well-known formula that expresses the survival function of the lifetime of a binary system in terms of its tail signature, under the condition that the component lifetimes are independent random variables, distributed according to a continuous distribution function $G(x)$. See also Remark 7 in Section 4.

By taking into account relation (4), we see that the standard signature is obtained from the tail signature by

$$
s_{l, r}=\bar{S}_{l-1, r-1}-\bar{S}_{l, r-1}-\bar{S}_{l-1, r}+\bar{S}_{l, r},
$$

where $1 \leq l<r \leq n$.

Examples of tail signatures for special cases of three-state systems will be deferred to Section 4. For the moment, we restrict ourselves to the following remark, where an extreme situation is considered.

Remark 3. In the one-dimensional case, degenerate signatures have a very clear meaning: we have $s_{k}=1$ for some $1 \leq k \leq n$ if and only if the system is of $(n-k+1)$-out-of- $n$ type. The following analogous situation holds for bivariate signatures: $s_{h, k}=1$ for some pair $1 \leq h<k \leq n$ if and only if the system is in the up state until $n-h+1$ components are up, and falls to the complete down state as soon as exactly $k$ component failures are recorded (independently of the identity of the failed components). 
Let

$$
S_{n_{1}, n_{2}}=\mathrm{P}\left(M_{1} \leq n_{1}, M_{2} \leq n_{2}\right)
$$

denote the coefficients of the cumulative (bivariate) signature. A formula similar to (5) can be given for the cumulative probabilities $\mathrm{P}\left(\Upsilon_{1} \leq \xi_{1}, \Upsilon_{2} \leq \xi_{2}\right)$ in terms of the coefficients $S_{n_{1}, n_{2}}$.

Remark 4. Provided that we maintain the condition that the components are binary, all the above notions and results extend quite easily to the general case of a coherent system with $v>3$ states. In such a case the signature is the discrete density function $s$ of $d=v-1,\{1, \ldots, n\}$ valued, ordered random variables $M_{1}, \ldots, M_{d}$, the anchors. The reliability function of interest is related to the joint survival function $\bar{F}$ of the continuous random variables $\Upsilon_{1}, \ldots, \Upsilon_{d}$, the calendar times to the different levels of degradation. An appropriate extension of Theorem 1 would deal with the connection between $\bar{F}$ and $s$. It is clear however that all the formulae become more and more involved as $v$ increases.

\subsection{Combinatorial meaning of $\bar{S}_{n_{1}, n_{2}}$}

Different aspects related to the combinatorial interpretations of univariate signatures have been analyzed in the literature; see, in particular, [2], [3], [7], and [10].

A significant interpretation for the coefficients of the term $u^{n_{1}} v^{n_{2}-n_{1}} z^{n-n_{2}}$ appearing in polynomial (5) can be given as well. Denote by $\{1,2, \ldots, n\}$ the ordered set of system components.

To any permutation $\pi$ of $\{1,2, \ldots, n\}$ (i.e. $\pi \in \mathcal{P}_{n}$ ), we associate the sets $\Sigma_{1}(\pi)$ and $\Sigma_{2}(\pi)$, which satisfy the following properties.

- $\Sigma_{1}$ and $\Sigma_{2}$ are subsets of $\{1,2, \ldots, n\}$ such that $\Sigma_{1} \subset \Sigma_{2}$.

- The elements of $\Sigma_{1}$ coincide exactly with the first $\left|\Sigma_{1}\right|$ elements of $\pi$ and the elements of $\Sigma_{2}$ coincide exactly with the first $\left|\Sigma_{2}\right|$ elements of $\pi$.

- If all the elements of $\Sigma_{1}$ are down and the remaining elements are $u p$, the system is in state 2 .

- If all the elements of $\Sigma_{2}$ are down and the remaining elements are up, the system is not in state 0 , i.e. it is in state 2 or 1 .

In other words, the complement of $\Sigma_{1}$ is a path set for the perfection state, and the complement of $\Sigma_{2}$ is a path set for the working system (perfection state or partial failure state).

Once the structure $\varphi$ has been fixed, the pair $\left(\Sigma_{1}(\pi), \Sigma_{2}(\pi)\right)$ is uniquely determined by the permutation $\pi$. In this respect, we claim that the quantity

$$
\frac{n !}{n_{1} !\left(n_{2}-n_{1}\right) !\left(n-n_{2}\right) !} \bar{S}_{n_{1}, n_{2}}
$$

equals the number of permutations $\pi$ such that

$$
\left|\Sigma_{1}(\pi)\right|=n_{1}, \quad\left|\Sigma_{2}(\pi)\right|=n_{2} .
$$

An example will be presented in Section 4. 


\section{The case of G-series and G-parallel systems of three-state modules}

In this section we consider two different three-stage structures, namely,

$$
\varphi_{A}:\{0,1\}^{n_{A}} \rightarrow\{0,1,2\}, \quad \varphi_{B}:\{0,1\}^{n_{B}} \rightarrow\{0,1,2\},
$$

and the 'series structure' $\varphi:\{0,1\}^{N} \rightarrow\{0,1,2\}$ defined by

$$
\begin{gathered}
N=n_{A}+n_{B}, \\
\varphi\left(a_{1}, \ldots, a_{n_{A}} ; b_{1}, \ldots, b_{n_{B}}\right)=\min \left(\varphi_{A}(a), \varphi_{B}(b)\right) .
\end{gathered}
$$

We call this system the $G$-series of $A$ and $B$, where $G$ stands for 'generalized'.

Structures of this type can be found in the literature on multistate systems; see, for example [13, Example 1.1, p. 19 and Section 4.2.1, p. 166]. Here we deal with the topic of their bivariate signatures.

Denote by $\bar{S} \equiv\left(\bar{S}_{n_{1}, n_{2}}\right), \bar{S}^{(A)} \equiv\left(\bar{S}_{n_{1}, n_{2}}^{(A)}\right)$, and $\bar{S}^{(B)} \equiv\left(\bar{S}_{n_{1}, n_{2}}^{(B)}\right)$ the tail signatures of $\varphi, \varphi_{A}$, and $\varphi_{B}$, respectively. In what follows we obtain $\bar{S}$ and show that it depends only on the pair $\left(\bar{S}^{(A)}, \bar{S}^{(B)}\right)$. To this end, with obvious notation, we argue as follows. We consider the calendar times to degradation steps for the G-series, given by

$$
\Upsilon_{1}=\min \left(\Upsilon_{1}^{(A)}, \Upsilon_{1}^{(B)}\right) \text { and } \Upsilon_{2}=\min \left(\Upsilon_{2}^{(A)}, \Upsilon_{2}^{(B)}\right)
$$

Then we write, for fixed $0<\xi_{1}<\xi_{2}$,

$$
\mathrm{P}\left(\Upsilon_{1}>\xi_{1}, \Upsilon_{2}>\xi_{2}\right)=\mathrm{P}\left(\Upsilon_{1}^{(A)}>\xi_{1}, \Upsilon_{1}^{(B)}>\xi_{1}, \Upsilon_{2}^{(A)}>\xi_{2}, \Upsilon_{2}^{(B)}>\xi_{2}\right) .
$$

We assume that the lifetimes $T_{1}, \ldots, T_{n_{A}}, T_{1}^{\prime}, \ldots, T_{n_{B}}^{\prime}$ are i.i.d. random variables, distributed according to $G(t)$. Therefore,

$$
\mathrm{P}\left(\Upsilon_{1}>\xi_{1}, \Upsilon_{2}>\xi_{2}\right)=\mathrm{P}\left(\Upsilon_{1}^{(A)}>\xi_{1}, \Upsilon_{2}^{(A)}>\xi_{2}\right) \mathrm{P}\left(\Upsilon_{1}^{(B)}>\xi_{1}, \Upsilon_{2}^{(B)}>\xi_{2}\right)
$$

From this product, and by using (5) for each of the two factors on the right-hand side, we obtain an expression for $\mathrm{P}\left(\Upsilon_{1}>\xi_{1}, \Upsilon_{2}>\xi_{2}\right)$ as a homogeneous polynomial of degree $N=n_{A}+n_{B}$ in the variables $u, v$, and $z$. We also obtain a different expression for $\mathrm{P}\left(\Upsilon_{1}>\xi_{1}, \Upsilon_{2}>\xi_{2}\right)$ by directly applying (5) to the structure $\varphi$. This latter expression will still have the form of a homogeneous polynomial of degree $N$ in the variables $u, v$, and $z$, but the polynomial coefficients will be expressed in terms of the quantities $\bar{S}_{h, k}$ related to the tail signature of $\varphi$.

The expressions for $\bar{S}_{h, k}$ in terms of $\bar{S}^{(A)}$ and $\bar{S}^{(B)}$ are obtained by comparing the two polynomial forms term by term. We give the formulae in the following result, under the common convention that a binomial coefficient $\left(\begin{array}{l}v \\ u\end{array}\right)$ is zero whenever $u<0$ or $u>v$.

Theorem 2. For $0 \leq n_{1}<n_{2} \leq N$, we have

$$
\bar{S}_{n_{1}, n_{2}}=\sum_{a_{1}=0}^{n_{A}-1} \sum_{a_{2}=a_{1}+1}^{n_{A}} \bar{S}_{a_{1}, a_{2}}^{(A)} \bar{S}_{n_{1}-a_{1}, n_{2}-a_{2}}^{(B)} \frac{\left(\begin{array}{l}
n_{1} \\
a_{1}
\end{array}\right)\left(\begin{array}{c}
n_{2}-n_{1} \\
a_{2}-a_{1}
\end{array}\right)\left(\begin{array}{c}
N-n_{2} \\
n_{A}-a_{2}
\end{array}\right)}{\left(\begin{array}{c}
N \\
n_{A}
\end{array}\right)} .
$$

Proof. As we have already mentioned, there is no loss of generality as far as the computation of the signatures of the different systems is concerned, if we assume that the lifetimes of all the components are i.i.d. with a continuous distribution function $G$. In such cases, by separately applying (5) to the terms

$$
\mathrm{P}\left(\Upsilon_{1}^{(A)}>\xi_{1}, \Upsilon_{2}^{(A)}>\xi_{2}\right) \quad \text { and } \quad \mathrm{P}\left(\Upsilon_{1}^{(B)}>\xi_{1}, \Upsilon_{2}^{(B)}>\xi_{2}\right)
$$


the right-hand side of (6) becomes

$$
\begin{aligned}
& \sum_{a_{1}=0}^{n_{A}-1} \sum_{a_{2}=a_{1}+1}^{n_{A}} u^{a_{1}} v^{a_{2}-a_{1}} z^{n_{A}-a_{2}} \bar{S}_{a_{1}, a_{2}}^{(A)} \frac{n_{A} !}{a_{1} !\left(a_{2}-a_{1}\right) !\left(n_{A}-a_{2}\right) !} \\
& \times \sum_{b_{1}=0}^{n_{B}-1} \sum_{b_{2}=b_{1}+1}^{n_{B}} u^{b_{1}} v^{b_{2}-b_{1}} z^{n_{B}-b_{2}} \bar{S}_{b_{1}, b_{2}(B)} \frac{n_{B} !}{b_{1} !\left(b_{2}-b_{1}\right) !\left(n_{B}-b_{2}\right) !} .
\end{aligned}
$$

By directly applying (5) to the structure $\varphi$, the left-hand side of (6) becomes

$$
\sum_{n_{1}=0}^{N-1} \sum_{n_{2}=n_{1}+1}^{N} u^{n_{1}} v^{n_{2}-n_{1}} z^{N-n_{2}} \bar{S}_{n_{1}, n_{2}} \frac{N !}{n_{1} !\left(n_{2}-n_{1}\right) !\left(N-n_{2}\right) !} .
$$

Now we consider all the terms resulting from the product of the two sums in (8). We fix a pair $\left(n_{1}, n_{2}\right)$ with $0 \leq n_{1}<n_{2} \leq N$ and collect all the terms that are of degree $n_{1}$ in the variable $u$ and of degree $n_{2}$ in the variable $v$; namely, we collect all the terms deriving from the conditions

$$
a_{1}+b_{1}=n_{1}, \quad a_{2}+b_{2}=n_{2} .
$$

The sum of all such terms then takes the form

$$
\sum_{a_{1}=0}^{n_{A}-1} \sum_{a_{2}=a_{1}+1}^{n_{A}} \Delta\left(a_{1}, a_{2}\right) \bar{S}_{a_{1}, a_{2}}^{(A)} \bar{S}_{n_{1}-a_{1}, n_{2}-a_{2}}^{(B)} u^{n_{1}} v^{n_{2}-n_{1}} z^{N-n_{2}},
$$

where

$$
\begin{aligned}
\Delta\left(a_{1}, a_{2}\right):= & \mathbf{1}_{\left\{0 \leq n_{1}-a_{1} \leq n_{B}-1\right\}} \times \mathbf{1}_{\left\{n_{1}-a_{1}+1 \leq n_{2}-a_{2} \leq n_{B}\right\}} \\
& \times \frac{n_{A} !}{a_{1} !\left(a_{2}-a_{1}\right) !\left(n_{A}-a_{2}\right) !} \\
& \times \frac{n_{B} !}{\left(n_{1}-a_{1}\right) !\left(n_{2}-n_{1}-\left(a_{2}-a_{1}\right)\right) !\left(n_{B}-\left(n_{2}-a_{2}\right)\right) !} .
\end{aligned}
$$

For this reason, (8) can be rewritten as

$$
\sum_{n_{1}=0}^{N-1} \sum_{n_{2}=n_{1}+1}^{N} \sum_{a_{1}=0}^{n_{A}-1} \sum_{a_{2}=a_{1}+1}^{n_{A}} \Delta\left(a_{1}, a_{2}\right) \bar{S}_{a_{1}, a_{2}}^{(A)} \bar{S}_{n_{1}-a_{1}, n_{2}-a_{2}}^{(B)} u^{n_{1}} v^{n_{2}-n_{1}} z^{N-n_{2}} .
$$

By comparing the terms containing the monomials $u^{n_{1}} v^{n_{2}-n_{1}} z^{N-n_{2}}$ in (8) and (9), we obtain

$$
\bar{S}_{n_{1}, n_{2}} \frac{N !}{n_{1} !\left(n_{2}-n_{1}\right) !\left(N-n_{2}\right) !}=\sum_{a_{1}=0}^{n_{A}-1} \sum_{a_{2}=a_{1}+1}^{n_{A}} \Delta\left(a_{1}, a_{2}\right) \bar{S}_{a_{1}, a_{2}}^{(A)} \bar{S}_{n_{1}-a_{1}, n_{2}-a_{2}}^{(B)},
$$

whence (7) immediately follows.

We can define the G-parallel of the two structures $\varphi_{A}$ and $\varphi_{B}$ by letting

$$
\varphi\left(a_{1}, \ldots, a_{n_{A}} ; b_{1}, \ldots, b_{n_{B}}\right)=\max \left(\varphi_{A}(a), \varphi_{B}(b)\right) .
$$

An analogue of Theorem 2 for the cumulative signature of $\varphi$ can easily be given. 
Remark 5. The proof of Theorem 2 is based on the product formula (6) and the repeated use of (5). The latter equation has a basic role in the derivation of the bivariate signatures of G-series and G-parallel systems. This argument parallels the development that we used in [10] concerning the signature of binary systems obtained as aggregations of separate modules. See also Remark 8 in Section 4. On the other hand, (5) can have an important role in showing that several existing results about one-dimensional signatures can be extended to the twodimensional case.

Remark 6. In the G-series system we have $\Upsilon_{1}=\Upsilon_{1}^{(A)} \wedge \Upsilon_{1}^{(B)}$ and $\Upsilon_{2}=\Upsilon_{2}^{(A)} \wedge \Upsilon_{2}^{(B)}$. Note that no similar 'minimum-type' relation holds in general for the anchors $M_{1}$ and $M_{2}$. In other words, we generally have

$$
M_{1} \neq \min \left(M_{1}^{(A)}, M_{1}^{(B)}\right), \quad M_{2} \neq \min \left(M_{2}^{(A)}, M_{2}^{(B)}\right) .
$$

It can be clarifying in this respect to consider the special case where $A$ and $B$ have degenerate (bivariate) signatures (see Remark 3). By applying Theorem 2 we see that, while the distributions of $\min \left(M_{1}^{(A)}, M_{1}^{(B)}\right)$ and $\min \left(M_{2}^{(A)}, M_{2}^{(B)}\right)$ are degenerate, the signature of the $\mathrm{G}$-series of $A$ and $B$ is not. See also Remark 10 in Section 4.

\section{Discussion and concluding remarks}

As already mentioned, further aspects of bivariate or, generally, multivariate signatures are worth mentioning. We present two examples for illustration. In the first example we consider the combinatorial interpretation of the tail signature introduced in Section 1.

Example 1. Let us consider a bridge network with four nodes, $a, b, c, d$, and five edges, 1, 2, 3, 4, 5; see Figure 1. We assume that the nodes are completely reliable, whereas the edges are subject to failure (thus, $\{1,2,3,4,5\}$ is seen as the set of bridge components). For two given nodes $\alpha$ and $\beta$, the failure of the edge $(\alpha, \beta)$, incident to $\alpha$ and $\beta$, means the disruption of the connection between $\alpha$ and $\beta$ along this edge. State 2, complete perfection, for this system corresponds to the state where all the nodes are connected to each other. State 1, partial failure, for this system corresponds to the case where the nodes are separated into two nonconnected sets. State 0, complete failure, for this system corresponds to the case where the nodes are separated into more than two nonconnected sets. For example, if edges 1 and 2 fail, there will be two nonconnected sets of nodes, $\{a\}$ and $\{b, c, d\}$. If, in addition, edges 4 and 5 fail, we will have three separated sets of nodes, $\{a\},\{b\}$, and $\{c, d\}$.

Now consider the process of destruction. State 0 appears at the fourth step of destruction. State 1 appears at either the second or third step. It appears at the second step if and only if the

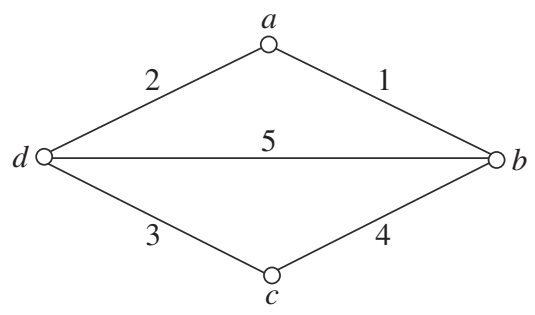

FIGURE 1: Bridge network. 
first two edges to fail are 1 and 2 or 3 and 4 . Whence

$$
s_{2,4}=\frac{1}{5}, \quad s_{3,4}=\frac{4}{5} .
$$

It is easy to check that $\bar{S}_{n_{1}, n_{2}}=1$ if and only if $\left(n_{1}, n_{2}\right)$ coincides with one of the following pairs: $(0,1),(0,2),(0,3),(1,2),(1,3)$. For $\left(n_{1}, n_{2}\right)=(2,3)$, we have $\bar{S}_{n_{1}, n_{2}}=\frac{4}{5}$. For all the remaining $\left(n_{1}, n_{2}\right)$ pairs, we have $\bar{S}_{n_{1}, n_{2}}=0$.

For $n_{1}=2$ and $n_{2}=3$, we obtain, as the coefficient of the term $u^{2} v^{1} z^{2}$,

$$
\frac{4}{5} \frac{5 !}{2 ! 1 ! 2 !}=24 \text {. }
$$

Excepting the two 'triangles' $(1,2,5)$ and $(3,4,5)$, the remaining sets of three edges, each being up, determine the up state for the system. Therefore, there are eight path sets, each consisting of three edges, for the up state. For each such set, there may be three different pairs of edges on the first two positions. In total, we have $24=8 \times 3$ pairs of path sets with the desired properties.

In the next example, we apply Theorem 2 to a pair of simple three-state systems.

Example 2. Consider two systems $A$ and $B$, with $n_{A}=n_{B}=4$ components, defined by

$$
\begin{aligned}
& \varphi_{A}(x)=\max \left(x_{1}+\max \left(x_{2}, x_{3}\right)+x_{4}-1,0\right), \\
& \varphi_{B}(x)=\max \left(x_{1}+\min \left(x_{2}, x_{3}\right)+x_{4}-1,0\right) .
\end{aligned}
$$

Both structures take values in the set $\{0,1,2\}$, are monotone, and satisfy the regularity condition in (1). Their signatures are given by

$$
\begin{aligned}
& \mathrm{P}\left(M_{1}^{(A)}=1, M_{2}^{(A)}=2\right)=\frac{1}{6}, \\
& \mathrm{P}\left(M_{1}^{(A)}=1, M_{2}^{(A)}=3\right)=\frac{1}{3}, \\
& \mathrm{P}\left(M_{1}^{(A)}=2, M_{2}^{(A)}=3\right)=\frac{1}{2} ;
\end{aligned}
$$

furthermore,

$$
\begin{aligned}
& \mathrm{P}\left(M_{1}^{(B)}=1, M_{2}^{(B)}=2\right)=\frac{2}{3}, \\
& \mathrm{P}\left(M_{1}^{(B)}=1, M_{2}^{(B)}=3\right)=\frac{1}{3} .
\end{aligned}
$$

The claim that $\mathrm{P}\left(M_{1}^{(A)}=1, M_{2}^{(A)}=2\right)=\frac{1}{6}$ follows from the fact that the event $\left(M_{1}^{(A)}=1\right.$, $M_{2}^{(A)}=2$ ) means that the first two failures involve components 1 and 4 , and the third and fourth failures involve components 2 and 3 . Of the $4 !=24$ permutations of $\{1,2,3,4\}$, exactly $24 / 2$ ! 2 ! satisfy this condition. Similarly, the event $\left(M_{1}^{(A)}=2, M_{2}^{(A)}=3\right)$ means that a permutation starting with component 2 or 3 will occur, with exactly 12 permutations satisfying this condition. Thus,

$$
\bar{S}_{0,2}^{(A)}=\frac{5}{6}, \quad \bar{S}_{1,2}^{(A)}=\frac{1}{2}, \quad \bar{S}_{0,2}^{(B)}=\frac{1}{3} .
$$

All other tail probabilities equal 0 , apart from $\bar{S}_{0,1}^{(A)}$ and $\bar{S}_{0,1}^{(B)}$, which are always equal to 1 . For the special case $n_{A}=n_{B}=4$, (7) reads

$$
\bar{S}_{n_{1}, n_{2}}=\sum_{a_{1}=0}^{3} \sum_{a_{2}=a_{1}+1}^{4} \bar{S}_{a_{1}, a_{2}}^{(A)} \bar{S}_{n_{1}-a_{1}, n_{2}-a_{2}}^{(B)} \frac{\left(\begin{array}{l}
n_{1} \\
a_{1}
\end{array}\right)\left(\begin{array}{l}
n_{2}-n_{1} \\
a_{2}-a_{1}
\end{array}\right)\left(\begin{array}{l}
8-n_{2} \\
4-a_{2}
\end{array}\right)}{\left(\begin{array}{l}
8 \\
4
\end{array}\right)} .
$$

Now fix a pair $\left(n_{1}, n_{2}\right)$ with $1 \leq n_{1}<n_{2}<7$. We see that, in order to compute $\bar{S}_{n_{1}, n_{2}}$, we must consider only the cases with $n_{1}-a_{1}=0, n_{2}-a_{2}=1$, and $n_{1}-a_{1}=0, n_{2}-a_{2}=2$. 
We conclude the paper with some general remarks.

Remark 7. (Marginal signatures.) The anchors $M_{1}$ and $M_{2}$ are such that $\mathrm{P}\left(M_{1}<M_{2}\right)=1$. Then the marginal survival function of $M_{2}$ can be immediately obtained from the (bivariate) tail signature $\bar{S}$ by letting

$$
\bar{S}_{r}^{(2)}:=\mathrm{P}\left(M_{2}>r\right)=\bar{S}_{0, r}
$$

for $r=1, \ldots, n-1$. Similarly, the marginal survival function of $M_{1}$ can be obtained from the (bivariate) cumulative signature $S$ by letting

$$
S_{l}^{(1)}:=\mathrm{P}\left(M_{1} \leq l\right)=S_{l, n}
$$

for $l=1, \ldots, n-1$.

The meaning of the marginal distribution of, say, $M_{2}$ is clear: it is the (one-dimensional) signature of the binary system that fails when our three-state system reaches complete failure, state 0 , and it is $u p$ until our system remains in perfection, state 2 , or in partial failure, state 1 . The meaning of the marginal distribution of $M_{1}$ is similar. See also [8] and [9] for discussions and examples about marginal one-dimensional signatures of multistate coherent systems.

Let us now consider the random variable $\Upsilon_{2}$ defined in (3). For the same binary system defined as above, $\Upsilon_{2}$ can be seen, in its turn, as the (calendar) time to failure. The marginal survival function of $\Upsilon_{2}$ is given by

$$
\mathrm{P}\left(\Upsilon_{2}>\xi\right)=\mathrm{P}\left(\Upsilon_{1}>0, \Upsilon_{2}>\xi\right) .
$$

By letting $\xi_{1}=0, \xi_{2}=\xi$, and $n_{2}=k$ in (5) and by taking into account (10), we obtain

$$
\mathrm{P}\left(\Upsilon_{2}>\xi\right)=\sum_{k=1}^{n}\left(\begin{array}{l}
n \\
k
\end{array}\right) v^{k}(1-v)^{n-k} \bar{S}_{k}^{(2)},
$$

where $v=G(\xi)$. We have thus re-obtained, as a special application of (5), the formula that relates the reliability function of a coherent binary system to its tail signature.

Remark 8. Consider (7), and set $n_{1}=0$ and $n_{2}=n$. Then

$$
\bar{S}_{0, n}=\sum_{a_{2}=1}^{n_{A}} \frac{\left(\begin{array}{c}
n \\
a_{2}
\end{array}\right)\left(\begin{array}{c}
N-n \\
n_{A}-a_{2}
\end{array}\right)}{\left(\begin{array}{c}
N \\
n_{A}
\end{array}\right)} \bar{S}_{0, a_{2}}^{(A)} \bar{S}_{0, n-a_{2}}^{(B)} .
$$

Now let

$$
\bar{S}_{r}^{(2)}:=\bar{S}_{0, r}, \quad \bar{S}_{a}^{(A, 2)}:=\bar{S}_{0, a}^{(A)}, \quad \bar{S}_{b}^{(B, 2)}:=\bar{S}_{0, b}^{(B)} .
$$

Thus, (11) becomes

$$
\bar{S}_{r}^{(2)}=\sum_{a=1}^{n_{A}} \frac{\left(\begin{array}{c}
r \\
a
\end{array}\right)\left(\begin{array}{c}
N-r \\
n_{A}-a
\end{array}\right)}{\left(\begin{array}{c}
N \\
n_{A}
\end{array}\right)} \bar{S}_{a}^{(A, 2)} \bar{S}_{n-a}^{(B, 2)} .
$$

In view of the arguments in Remark $7, \bar{S}_{a}^{(A, 2)}$ and $\bar{S}_{b}^{(B, 2)}$ are tail signatures of the two different binary systems, respectively obtained from the systems $\varphi_{A}$ and $\varphi_{B}$. By the same token, $\bar{S}_{r}^{(2)}$ can be seen as the tail signature of the binary system obtained from the G-series of $\varphi_{A}$ and $\varphi_{B}$.

Similarly to in Remark 7, we can conclude as follows. In [10] we presented an explicit formula for the (one-dimensional) tail signature of the system defined as the series of two separate binary systems. Here, such a formula has been re-obtained by means of a special application of (7). A different approach to the computation of signatures of binary systems composed of separate modules has been presented in [4]. 
Remark 9. Bivariate discrete distributions whose marginals are one-dimensional signatures of coherent binary systems also emerge in the study of two correlated binary systems, as developed in [14]. Generally, such distributions are, however, not two-dimensional signatures in the sense considered here.

Remark 10. Consider a system with $v$ possible states and anchors $M_{1}, \ldots, M_{v-1}$. Here we let $v=3$, but this remark is also valid for $v=2$ or any $v>3$. Let us moreover think of the cases when the subsequent failures of the components are not observable (only the system failure is observable) or when at least the identities of failed components are not observable. In such cases, it is interesting to interpret the pair $\left(M_{1}, M_{2}\right)$ as an internal clock: $\left(M_{1}, M_{2}\right)$ may be only partially observable, but it constitutes the basic element that determines the failure of the system. Furthermore, we can interpret the pair of degradation times $\left(\Upsilon_{1}, \Upsilon_{2}\right)$ as a phenomenological clock. Of course, the relation between $\left(M_{1}, M_{2}\right)$ and $\left(\Upsilon_{1}, \Upsilon_{2}\right)$ is not 'deterministic'. However, the correlation between the two pairs is rather strong; in fact, the conditional distribution of $\left(\Upsilon_{1}, \Upsilon_{2}\right)$ given $\left(M_{1}, M_{2}\right)$, which was dealt with in Section 1, shows that, in some suitable sense, $\left(\Upsilon_{1}, \Upsilon_{2}\right)$ is stochastically increasing with respect to $\left(M_{1}, M_{2}\right)$.

In reliability problems, it is important to clarify whether the phenomenological clock or the internal clock is used. The two approaches do not generally coincide.

In particular, when we form a supersystem by assembling two different (binary or multistate) structures $\varphi_{A}$ and $\varphi_{B}$, it is essential to clarify if we define the new system in terms of the corresponding pairs of anchors or degradation times. In Section 3 we defined the operation of G-series in terms of the pairs of degradation times (in this respect, see also Remark 6). We can imagine, however, situations where the observed times of subsequent failures are not relevant, but the number of failed components in each of the subsystems is relevant. Situations of this type are typical for network gradual disintegrations that result from component failures. An example is a series assembly of information transmission networks that are subject to unintentional attacks on their nodes; see [8, Section 2.4.2] and [12].

Remark 11. Consider a recurrent v-state system where an organizing structure admits, as supercomponents, $Q$ modules, with each module containing $R$ components. Thus, the system contains $Q R$ basic components. We suppose that the modules are separated, in the sense that each contains its own components and they are all similar, i.e. they share the same $v$-state structure.

It is possible to extend the argument presented in [10] about binary recurrent systems, based on a closure property of the class of homogeneous polynomials in $v=2$ variables, to this case. Such an extension allows us to conclude that the signature of the multistate recurrent system can be expressed in terms of the signature of the organizing structure and the signature of the modules. Generally, such an expression may be very complicated and, perhaps, of scarce practical use. However, such a result can be important from a theoretical point of view and shows a method to generate different systems sharing the same multivariate signatures.

\section{References}

[1] Barlow, R. E. and Proschan, F. (1975). Statistical Theory of Reliability and Life Testing. Holt, Rinehart and Winston, New York.

[2] Boland, P. J. (2001). Signatures of indirect majority systems. J. Appl. Prob. 38, 597-603.

[3] Boland, P. J., Samaniego, F. J. and Vestrup, E. M. (2003). Linking dominations and signatures in network reliability theory. In Mathematical and Statistical Methods in Reliability (Trondheim, 2002), World Scientific, River Edge, NJ, pp. 89-103.

[4] Da, G., Zheng, B. And Hu, T. (2012). On computing signatures of coherent systems. J. Multivariate Anal. 103, $142-150$. 
[5] Elperin, T., GertsbaKh, I. And Lomonosov, M. (1991). Estimation of network reliability using graph evolution models. IEEE Trans. Reliab. 40, 572-581.

[6] Gertsbakh, I. and Shpungin, Y. (2004). Combinatorial approaches to Monte Carlo estimation of network lifetime distribution. Appl. Stoch. Models Business Industry 20, 49-57.

[7] Gertsbakh, I. B. and Shpungin, Y. (2009). Models of Network Reliability: Analysis, Combinatorics, and Monte Carlo. CRC Press, Boca Raton, FL.

[8] Gertsbakh, I. And Shpungin, Y. (2011). Network Reliability and Resilience. Springer.

[9] Gertsbakh, I. and Shpungin, Y. (2012). Multidimensional spectra of multistate systems with binary components. In Recent Advances in System Reliability, eds A. Lisniansky and I. Frenkel, Springer, London, pp. 49-61.

[10] Gertsbakh, I., Shpungin, Y. and Spizzichino, F. (2011). Signatures of coherent systems built with separate modules. J. Appl. Prob. 48, 843-855.

[11] Kochar, S., Mukerjee, H. and Samaniego, F. J. (1999). The "signature" of a coherent system and its application to comparisons among systems. Naval Res. Logistics 46, 507-523.

[12] Levitin, G., Gertsbakh, I. and Shpungin, Y. (2011). Evaluating the damage associated with intentional network disintegration. Reliab. Eng. System Safety 96, 433-439.

[13] Lisnianski, A. And Levitin, G. (2003). Multi-State System Reliability. World Scientific, River Edge, NJ.

[14] Navarro, J., Samaniego, F. J. and Balakrishnan, N. (2010). The joint signature of coherent systems with shared components. J. Appl. Prob. 47, 235-253.

[15] Navarro, J., Samaniego, F. J., Balakrishnan, N. and Bhattacharaya, D. (2008). On the application and extension of system signatures in engineering reliability. Naval Res. Logistics 55, 314-326.

[16] Samaniego, F. J. (1985). On the closure of the IFR class under formation of coherent systems. IEEE Trans. Reliab. R-34, 69-72.

[17] Samaniego, F. J. (2007). System Signatures and Their Application in Engineering Reliability. Springer, New York.

[18] SpIzzichino, F. (2008). The role of signature and symmetrization for systems with non-exchangeable components. In Advances in Mathematical Modeling for Reliability, eds T. Bedford et al. , IOS Press, Amsterdam, pp. 138-148.

[19] Spizzichino, F. And Navarro, J. (2012). Signatures and symmetry properties of coherent systems. In Recent Advances in System Reliability, eds A. Lisniansky and I. Frenkel, Springer, London, pp. 33-48. 